\title{
Preparation of Chitosan-graft-poly(vinyl acetate) Copolymers and Their Adsorption of Copper Ion
}

\author{
Trong-Ming Don, Chia-Fong KInG, ${ }^{*}$ and Wen-Yen CHIU ${ }^{*} \dagger$ \\ Department of Chemical Engineering, Tamkang University, Taipei 25147, Taiwan, Republic of China \\ * Department of Chemical Engineering, National Taiwan University, Taipei 10617, Taiwan, Republic of China
}

(Received October 25, 2001; Accepted March 5, 2002)

\begin{abstract}
Chitosan-graft-poly(vinyl acetate) copolymers were prepared by a graft copolymerization with a redox initiator, cerium ammonium nitrate. Chitosan molecules not only participated in the reaction, but also served as a role of surfactant. As a result, a stable dispersion solution was observed during the reaction. After drying, particulate membranes were formed because of the rigid chitosan chains at particles' surface. When the membranes were soaked in the water, the hydrophilic chitosan absorbed a great amount of water, but not the hydrophobic poly(vinyl acetate) chains mainly constituted in the core of particles. A two-stage behavior was observed during the adsorption of copper ion in the copolymer membrane. The first stage was governed by both the diffusion and reaction; whereas only diffusion dominated the second stage. The diffusion rate increased with an increase in the amount of chitosan. In order to increase its mechanical strength and acid resistance, the copolymer membrane was cross-linked with a glutaraldehyde. However, the specific adsorption quantity of copper ion decreased as the extent of cross-linking was increased.

KEY WORDS Chitosan / Poly(vinyl acetate)/ Graft Copolymer / Particulate Membrane /

Adsorption / Copper Ion /
\end{abstract}

Hybridization of natural polymers with synthetic polymers is of great interest because of its application to biomedical and biodegradable materials. ${ }^{1}$ The modification of natural polymers can improve their properties including toughness and water resistance. One of the natural polymers that draw great attention recently is chitosan. Chitosan is a derivative of chitin and is generally prepared by the partial deacetylation of chitin in a hot alkali solution. Chitin can be found in the skeletal materials of crustaceans and insects, and the cell walls of bacteria and fungi. The chain structure of chitin and chitosan is composed of $\beta$ - $(1,4)$ linked D-glucosamine and $N$-acetyl-D-glucosamine. Chitin is hardly soluble in any solvent, but chitosan can be dissolved in an acid solution and becomes a cationic polymer because of the protonation of amino groups at the $\mathrm{C}-2$ position of pyranose ring. Compared with synthetic polymers, chitosan has several important advantages, including biocompatibility, biodegradability and no toxicity. In addition, it has a great number of hydroxyl and amino groups on the pyranose rings. Therefore, chitosan is known as an excellent adsorbent for metal ions, most effectively in the uptake of transition metals including cadmium, copper, lead, uranium, mercury and chromium. ${ }^{2-5}$ The adsorbent capability of chitosan is generally attributed to the complex formation or chelating between the amino groups of the chitosan and the metal ions.

However, chitosan is a brittle material and can absorb a great amount of moisture. Therefore, it would be de- sired to improve its toughness and water resistance, for example, by a cross-linking of the chitosan chains with glutaraldehyde. ${ }^{6}$ However, another route was adopted in this work, which a hybrid copolymer was synthesized by grafting a synthetic polymer onto the chitosan chain. ${ }^{7}$ In this way, a new material with desired properties can be prepared by the chemical combination of natural and synthetic polymers. A strong redox initiator, ceric ion $\left(\mathrm{Ce}^{4+}\right)$, was used to oxidize the pyranose rings of polysaccharide and produce free radicals on them. Two kinds of initiation reaction have been proposed in the literature. ${ }^{8-13}$ One is the direct abstraction of hydrogen atom from the carbon having a hydroxyl group. The following oxidation of this hydrogen atom by ceric ion $\left(\mathrm{Ce}^{4+}\right)$ yields a proton, cerous ion $\left(\mathrm{Ce}^{3+}\right)$ and a free radical on the carbon. The other route is that the ceric ion $\left(\mathrm{Ce}^{4+}\right)$ forms a complex with the nucleophilic groups such as $\mathrm{NH}_{2}$ and $\mathrm{OH}$ at $\mathrm{C}-2$ carbon and $\mathrm{C}-3$ carbon, respectively. Subsequently, the $\mathrm{C}-\mathrm{C}$ bond between the functional groups cleaves to yield an aldehyde and a free radical. Chen ${ }^{14}$ has shown the evidence of the formation of an aldehyde where ceric ion was used to initiate the graft copolymerization of styrene onto starch. Once the initiation reaction has started, the graft copolymerization of monomer onto chitosan chains then can occur immediately following the traditional chain polymerization. Poly(vinyl acetate), PVAc, a leathery and water-resistant polymer, was chosen to improve the toughness of chitosan. The reaction mech-

${ }^{\dagger}$ To whom correspondence should be addressed (Tel: +886-2-23623259, Fax: +886-2-23623259, E-mail: ycchiu@ms.cc.ntu.edu.tw). 
anism and kinetics of the graft copolymerization of PVAc onto chitosan have been described in the previous article. ${ }^{7}$ In this article, the structures and metal ion uptake of chitosan-graft-poly(vinyl acetate) copolymers were examined.

\section{EXPERIMENTAL}

\section{Materials}

Chitosan (Tokyo Chemicals Inc., Tokyo) was purified before use. It was first dissolved into a $2 \%$ acetic acid solution, and then precipitated out by the addition of a concentrated $\mathrm{NaOH}$ solution. The precipitate was washed several times with a great amount of deionized water until it became neutral. The degree of deacetylation of chitosan was found to be $86 \%$ by a colloid titration method. ${ }^{15}$ In this method, a specific amount of chitosan-acetic acid solution was titrated with 0.0025 $\mathrm{N}$ PVSK (potassium polyvinylsulfate, $\left(\mathrm{C}_{2} \mathrm{H}_{3} \mathrm{O}_{4} \mathrm{SK}\right)_{n}$, $n=1500$ or above), where $0.1 \%$ toluidine blue was used as an indicator. The viscosity average molecular weight $\left(\bar{M}_{\mathrm{v}}\right)$ of chitosan, determined by a viscometric method at $25^{\circ} \mathrm{C}$, was found to be 616000 from the Mark-Houwink equation, $[\eta]=k \bar{M}_{\mathrm{v}}{ }^{\alpha}$, where $k$ and $\alpha$ were $1.38 \times 10^{-2}$ and 0.85 , respectively. ${ }^{16}$ Vinyl acetate (VAc) monomer from Acros organics (Belgium) was distilled under reduced pressure. Only the distillate obtained at the middle stage of distillation was used for polymerization. Cerium ammonium nitrate (CAN), $\mathrm{Ce}\left(\mathrm{NH}_{4}\right)_{2}\left(\mathrm{NO}_{3}\right)_{6}$, was a reagent-grade from Showa Chemical Inc. Copper (II) nitrate was a reagentgrade from Acros organics (Belgium). All the other chemicals were analytical grades or above and used as received without further purification.

\section{Graft Copolymerization}

The details of the graft copolymerization was described elsewhere. ${ }^{7}$ Only a brief description was shown here. $1.08 \times 10^{-2} \mathrm{~mol}$ of CAN initiator was added to the chitosan/acetic acid solution. The solution was heated to $60^{\circ} \mathrm{C}$ in an isothermal water bath and purged with nitrogen. Subsequently, $50 \mathrm{~g}$ of distilled VAc monomer was added to the $500 \mathrm{~mL}$ solution and the polymerization was immediately started. After $2 \mathrm{~h}$, a dispersion solution containing chitosan copolymer was obtained. The conversion $(X \%)$, grafting efficiency $(G E, \%)$ and grafting ratio (GR), are listed in Table . $^{7}$ The conversion was calculated with the following equation.

$$
X(\%)=\left(W_{1}-W_{\mathrm{CS}}\right) /\left(W_{\mathrm{VAc}}\right) \times 100
$$

In the equation, $W_{\mathrm{CS}}$ and $W_{\mathrm{VAc}}$ are the initial weight of chitosan and VAc monomer, respectively. $W_{1}$ is the final weight of drying product containing both the PVAc
Table I. The reaction conditions and the sample code of final samples

\begin{tabular}{lcccc}
\hline System & SE101 & SE105 & SE110 & SE115 \\
\hline $\mathrm{CS} / \mathrm{g}$ & 0.5 & 2.5 & 5 & 7.5 \\
$\mathrm{VAc} / \mathrm{g}$ & 50 & 50 & 50 & 50 \\
$X / \%^{\mathrm{a}}$ & 80.0 & 78.1 & 75.9 & 70.3 \\
$G E / \% \%^{\mathrm{b}}$ & 4.22 & 17.4 & 29.7 & 35.7 \\
$\mathrm{GR}^{\mathrm{c}}$ & 3.39 & 3.42 & 2.74 & 2.36 \\
\hline
\end{tabular}

${ }^{\mathrm{a}} X(\%)=$ (weight of total reacted monomer/initial monomer weight $) \times 100 .{ }^{\mathrm{b}} G E(\%)=$ (weight of grafted PVAc chains/weight of total monomer conversion) $\times 100 .{ }^{\mathrm{c}} \mathrm{GR}=$ (weight of grafted PVAc chains/weight of chitosan).

homopolymer and the chitosan-graft-PVAc copolymer. To calculate the grafting efficiency $(G E, \%)$ and grafting ratio (GR), acetone solvent was used to extract PVAc homopolymer from the drying product with a Soxhlet extractor. After $72 \mathrm{~h}$ of extraction, the remaining chitosan-graft-PVAc copolymer was dried and weighed $\left(W_{2}\right)$. The $G E(\%)$ and GR were calculated with the following equations.

$$
\begin{aligned}
G E(\%)= & (\text { weight of grafted PVAc chains } / \text { weight of } \\
& \text { total monomer conversion }) \times 100 \\
= & \left(W_{2}-W_{\mathrm{CS}}\right) /\left(W_{1}-W_{\mathrm{CS}}\right) \times 100 \\
\mathrm{GR}= & (\text { weight of grafted PVAc chains } / \text { weight } \\
& \text { of chitosan }) \\
= & \left(W_{2}-W_{\mathrm{CS}}\right) /\left(W_{\mathrm{CS}}\right)
\end{aligned}
$$

For the preparation of sample membrane, the dispersion solution was cast into a stainless mold and then dried at $50^{\circ} \mathrm{C}$ for $48 \mathrm{~h}$ with another $12 \mathrm{~h}$ in the vacuum oven at $80^{\circ} \mathrm{C}$.

\section{Cross-Linking of Chitosan-graft-poly(vinyl acetate) Copolymers}

Sample membranes of chitosan-graft-poly(vinyl acetate) copolymers were immersed into a $0.25 \%$ glutaraldehyde solution at $25^{\circ} \mathrm{C}$. After a period of time, the sample membranes were removed from the solution and washed with deionized water several times. They were then dried at $50^{\circ} \mathrm{C}$ for $48 \mathrm{~h}$ with another $12 \mathrm{~h}$ in the vacuum oven at $80^{\circ} \mathrm{C}$.

\section{Morphology Observation}

A scanning electron microscope (SEM, S-200 from Hitachi) was used to observe the morphology of sample membranes before and after cross-linking. Sample membranes in the wet state were also observed on the surface area. In this case, the membranes were first immersed in the deionized water for a period of time and then freeze-dried. 


\section{Copper Ion Adsorption}

Copper nitrate was used in the adsorption experiment and the adsorption of copper ion was carried out in a batch mode. The sample membrane was immersed into the copper ion solution with a concentration of $2000 \mathrm{ppm}$. The dimension of membrane is $20 \mathrm{~cm} \times$ $20 \mathrm{~cm} \times 0.2 \mathrm{~mm}$. The temperature was set at $25^{\circ} \mathrm{C}$ and the $\mathrm{pH}$ value was controlled at 4 . A small amount of solution was withdrawn periodically and filtered with a membrane filter $(0.45 \mu \mathrm{m})$. The filtrate was diluted into a suitable concentration range for the analysis of the residual copper ion with an atomic absorption spectrophotometer (Model 906AA, GBC Inc., USA).

\section{Mechanical Properties}

Tensile properties: ultimate tensile strength, initial modulus and elongation at break were measured using Universal Tensile Testing Instrument (RTM-1 from Yashima Works Co.). The tensile speed was $20 \mathrm{~mm}$ $\mathrm{min}^{-1}$. All the specimens were prepared from the sample membranes and had the dimension of $10 \times 1 \mathrm{~cm}$. Approximately 5 specimens were tested for each condition and the results were averaged.

\section{Swelling Studies}

Samples were immersed into the deionized water to observe the swelling behavior. The $\mathrm{pH}$ value of the water was controlled at 6 . After the excess water was removed with filter paper, the weight of swollen samples was measured at various time intervals. The procedure was repeated until there was no further weight increase. The degree of swelling was calculated by the weight of the swollen sample at a specific time divided by the sample weight in the dry state.

\section{RESULTS AND DISCUSSION}

\section{Preparation of Copolymer Membranes}

In this study, ceric ion $\left(\mathrm{Ce}^{4+}\right)$ was used to initiate the graft copolymerization of vinyl acetate (VAc) monomer onto the chitosan chains. The reaction system was a stable dispersion solution, and the monomer conversion was found to be between $70-80 \%$ after $2 \mathrm{~h}$ of reaction at $60^{\circ} \mathrm{C}$. After drying, SEM was used to observe the surface morphology of the membranes. Figure 1 shows that both of SE101 and SE115 are dense membranes. Yet, SE101 has a smooth surface; and with a greater amount of chitosan, SE115 becomes a particulate membrane. In this study, it is also important to examine the morphology changes of these membranes after soaking in the water, since the adsorption of copper ion was carried out in the water. Therefore, they were dipped inside the water for a period of time, until they were satu-

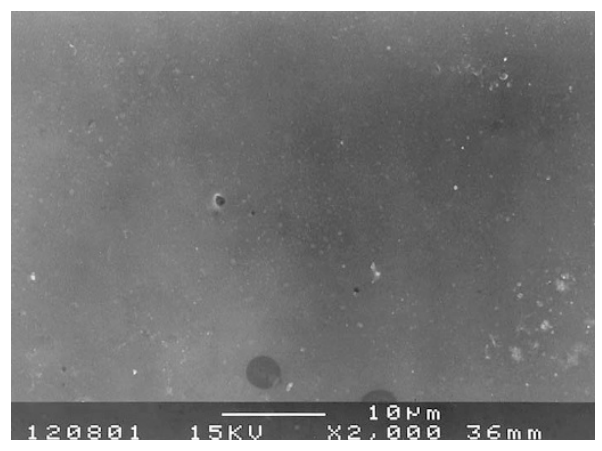

(a) SE011

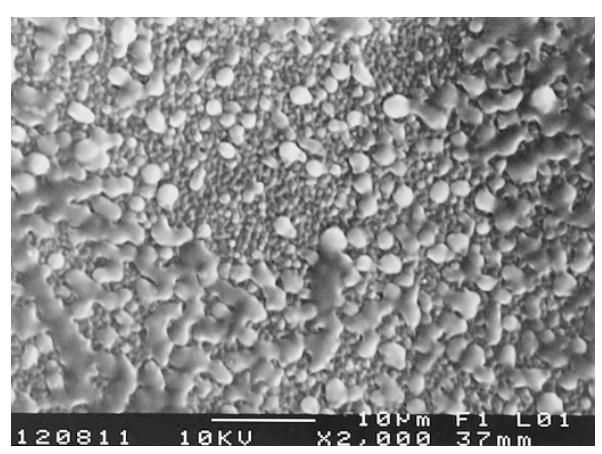

(b) SE115

Figure 1. SEM micrographs of the membranes' surface after oven drying.

rated with water. They were then taken out and freezedried. Figure 2 shows the surface morphology of these wet membranes. SE101 having the least amount of chitosan is still a dense membrane with a smooth surface. Yet, voids were observed for the SE115 membrane. As the amount of chitosan increased, the size of these voids also increased. Figure 3 shows the cross section of these wet membranes. The voids inside the membrane also increased in size with the chitosan amount. In addition, most particles were no longer discrete after immersion in water. They were connected together with bridges.

The reason that these membranes, especially with higher concentration of chitosan, are particulate membranes is that the reaction system is a stable dispersion solution and the surface of these dispersed particles is composed mainly of hydrophilic chitosan chains. Chitosan not only participates in the graft copolymerization, but also serves as a role of surfactant, which provided the stability of dispersion particles. The zeta potential of the dispersed particles at $\mathrm{pH} 6$ is $-54 \mathrm{mV}$ for a pure PVAc. Yet, with the addition of chitosan, it becomes a positive value, $12 \mathrm{mV}$, for a SE110 sample. This is attributed to the protonation of amino group in chitosan at the particle's surface, $\mathrm{NH}_{2} \rightarrow \mathrm{NH}_{3}{ }^{+}$. Furthermore, as the $\mathrm{pH}$ value decreases, the zeta potential of dispersed particles increases because more $\mathrm{NH}_{2}$ 


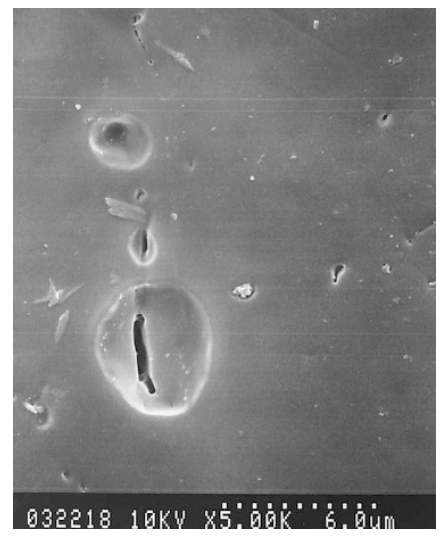

(a) SE101

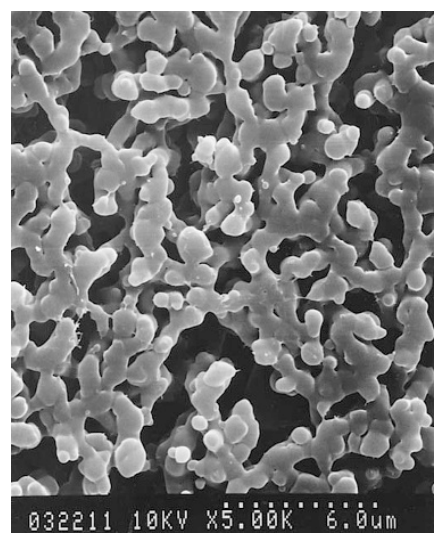

(c) SE110

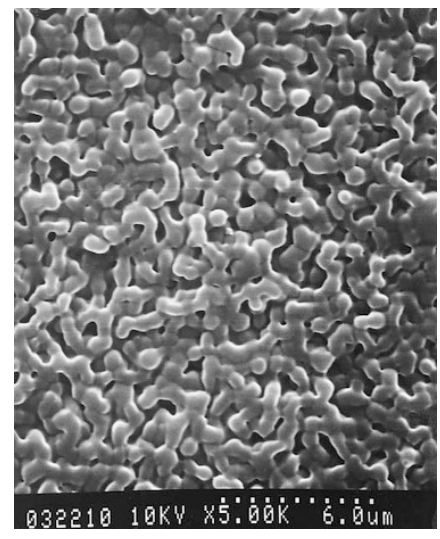

(b) SE105

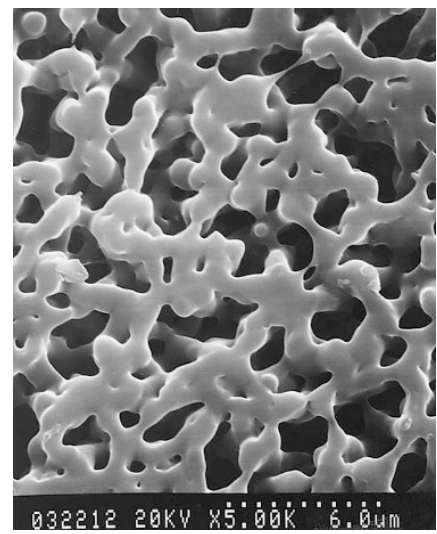

(d) SE115

Figure 2. SEM micrographs of the wet membranes' surface after freeze-drying.

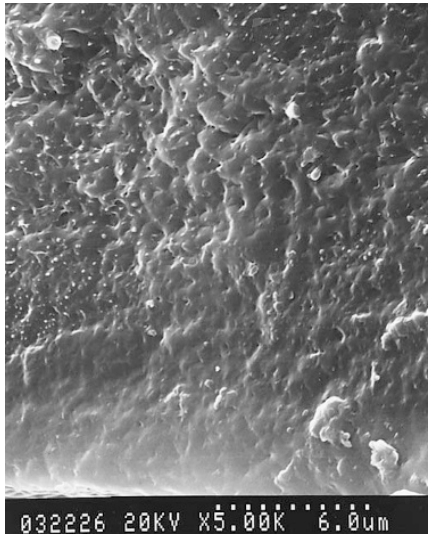

(a) SE101

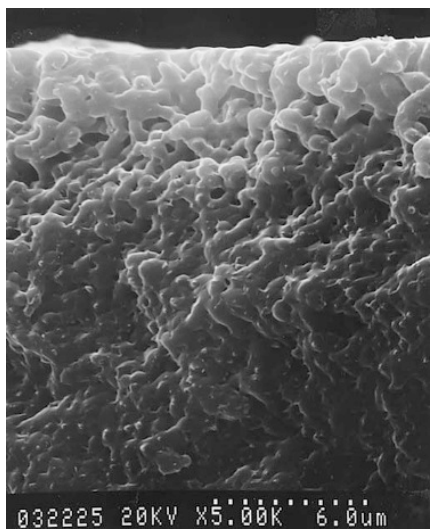

(b) SE105

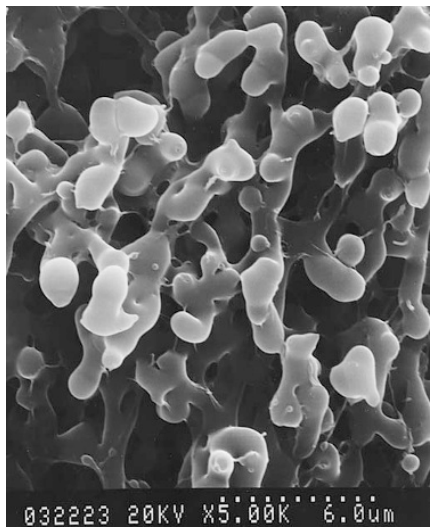

(c) SE115

Figure 3. SEM micrographs of the wet membranes' cross-section after freeze-drying.

groups are converted to the $\mathrm{NH}_{3}{ }^{+}$groups. On the contrary, the core of these particles consists of mostly hydrophobic poly(vinyl acetate), PVAc. The structure has also been proved in the last article with several analytical techniques. ${ }^{7}$ Therefore, when the dispersion solution was dried, the chitosan chains at the particles' surface were so rigid that they did not fuse together at the drying temperature. Consequently, they retained their particulate shape in the membrane. The size of these particles estimated from the SEM picture is in the range of 1-3 $\mu \mathrm{m}$. When the membrane was soaked in the water, the outer shell of particles swelled to a great extent because of the highly hydrophilic behavior of chitosan chains. The chitosan molecules were so relaxed in the water that they could diffuse and then intermingle with other chitosan molecules at nearby particles. In contrast, the hydrophobic PVAc core did not absorb much water. If the absorbed water was removed by freezedrying, voids were then left among the particles in the membrane. The size of voids increased as the capabil- 


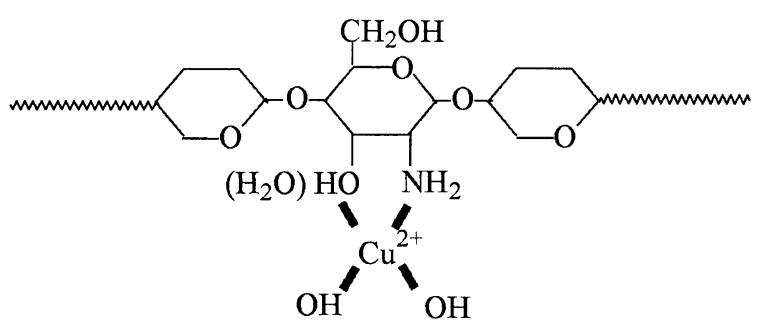

Scheme 1. The chelating of copper ion with chitosan.

ity of water absorption increased, which was directly proportional to the chitosan amount. The equilibrium degree of swelling increased from 2.6 for the SE101 sample to 11 for the SE115 sample. In addition, particles were connected together after freeze-drying, because of the fusion of the relaxed chitosan molecules in the water.

\section{Adsorption of Copper Ion}

There are many factors that can affect the adsorption capability of metal ions on the chitosan. These include the extent of protonation of amino group, the state and concentration of the metal ion in the solution, temperature and $\mathrm{pH}$ value of the solution and so on. It has been pointed out that the adequate solution acidity for the maximum adsorption of copper ion on chitosan is about $\mathrm{pH} 4 .^{5}$ Therefore, the $\mathrm{pH}$ was controlled at 4 and the temperature was set at $25^{\circ} \mathrm{C}$. At this state, copper ion exists as a free $\mathrm{Cu}^{2+}$ ion, and the adsorption of $\mathrm{Cu}^{2+}$ is through a chelating reaction ${ }^{17}$ as shown in the following scheme.

In this experiment, the adsorption rate is controlled by the following processes: ${ }^{4,18}$ (1) diffusion of the copper ion from the solution to the boundary film, (2) transport of the copper ion from the boundary film to the membrane surface, (3) transfer of the copper ion from the membrane surface to the chitosan-rich region including the bridges and outer shell of particles (shell diffusion), (4) transfer of the copper ion through the particles' shell into the PVAc-rich core of particles (core diffusion), (5) chelating reaction of the copper ion with the active sites. Processes (1) and (2) can be neglected when agitation is sufficient to avoid concentration gradients. The shell and core diffusion as well as the reaction are likely to be the rate controlling. If only the diffusion process is considered, Fick's second law can be applied as shown in eq 4 , where $C_{\mathrm{Cu}}$ is the concentration of copper ion at a specific time $(t)$ and the diffusion coefficient $(D)$ is assumed to be a constant. The concentration gradient of copper ion from the surface to the particle's interior thus can be obtained by solving the equation. Subsequently with the integration, one

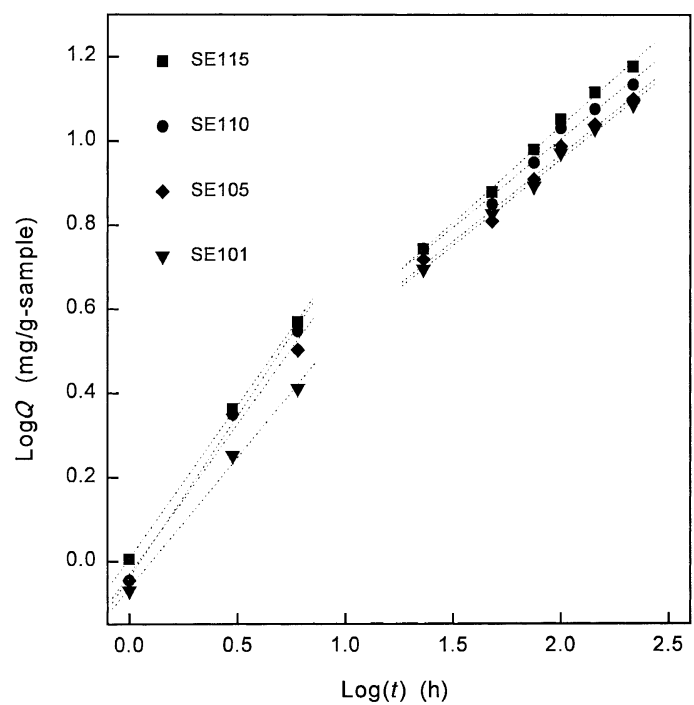

Figure 4. A Logarithmic plot of the specific adsorption quantity $Q$ of copper ion with time $t, Q=k t^{n}$. The maximum correlation coefficient $(R)$ values were obtained if two regression intervals were tested where one is from 0 to $6 \mathrm{~h}$ and the other is from 23 to $217 \mathrm{~h}$.

can obtain the relationship between the adsorption

$$
\frac{\partial C_{\mathrm{Cu}}}{\partial t}=D \nabla^{2} C_{\mathrm{Cu}}
$$

quantity of copper ion $(Q)$ with time. The integration shows that the adsorption quantity is proportional to the square root of time, $Q=k t^{1 / 2}$. This is the well-known solution of the Fick's second law. ${ }^{19}$ If a reaction occurs along with the diffusion process, the equation of continuity can be written as the following, where $R_{\mathrm{Cu}}$ represents the chelating reaction of the copper ion. This equation implies the dependence order of the adsorption quantity on the time would deviate from the one half.

$$
\frac{\partial C_{\mathrm{Cu}}}{\partial t}=D \nabla^{2} C_{\mathrm{Cu}}+R_{\mathrm{Cu}}
$$

Figure 4 is a logarithmic plot of the specific adsorption quantity versus time. A two-stage adsorption behavior was observed through a regression analysis of the equation $Q=k t^{n}$. Table II lists these $n$ values of various samples. They are in the range of 0.62 to 0.77 for the first stage, and 0.41 to 0.46 for the second stage. Generally, if $n$ value is close to one half, the diffusion process mainly controls the adsorption. It is thus presumed that both the diffusion and reaction in the first stage dominated the adsorption, whereas only diffusion controlled the second stage. From the previous SEM pictures, it is known that basically there are two regions in the particulate membrane. One is the chitosanrich region at the outer shell of particles and the other is the PVAc-rich region in the particles' core. As the membrane was immersed in the water, the chitosan-rich region absorbed a great amount of water and became 
Table II. $n$ Values from the regression equation $\left(Q=k t^{n}\right)$ and its correlation coefficient $R$ for various samples in the adsorption of copper ion

\begin{tabular}{ccccc}
\hline \multirow{2}{*}{ Sample } & \multicolumn{2}{c}{ First stage } & \multicolumn{2}{c}{ Second stage } \\
\cline { 2 - 5 } & $n$ & $R$ & $n$ & $R$ \\
\hline SE101 & 0.6225 & 0.998 & 0.4091 & 0.997 \\
SE105 & 0.7152 & 0.992 & 0.4107 & 0.993 \\
SE110 & 0.7268 & 0.998 & 0.4188 & 0.994 \\
SE115 & 0.7682 & 0.999 & 0.4576 & 0.998 \\
\hline
\end{tabular}

Two stages were fitted with the regression equation to obtain the maximum $R$-values.

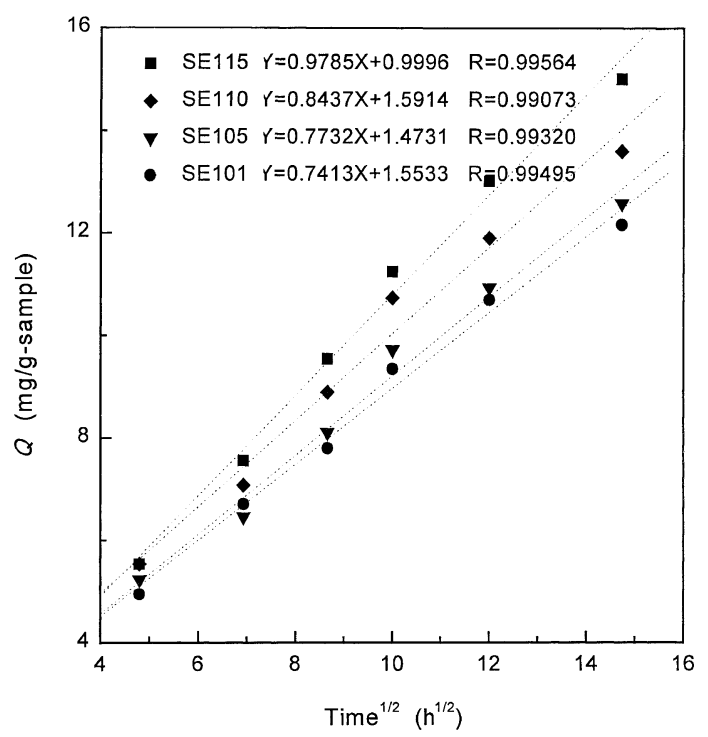

Figure 5. A linear plot of the specific adsorption quantity $Q$ of copper ion in the second stage with the square root of time $t$, $Q=k t^{1 / 2}$.

highly porous. Yet, the hydrophobic PVAc-rich core remained dense. Therefore, at the beginning of adsorption, copper ion diffuses easily to the active sites of particles' shell and bridges (shell diffusion) and then undergoes chelating reaction with the functional groups. The adsorption behavior at this stage is thus characterized by both the diffusion and the reaction. As the available sites on the particles' shell are occupied more and more, most of the copper ion cannot help to diffuse into the narrow pores inside the particles. It thus becomes diffusion control. A plot of the specific adsorption quantity in the second stage with the square root of time was obtained in Figure 5. The slope, directly proportional to the diffusion coefficient, increased with the chitosan amount added into the system.

\section{Cross-Linking with Glutaraldehyde}

The grafting of PVAc onto chitosan though improves the toughness and decreases the water absorption of dry membranes; yet, it does not give appreciable improvement on the mechanical strength of wet membranes. This is again because they are particulate mem-

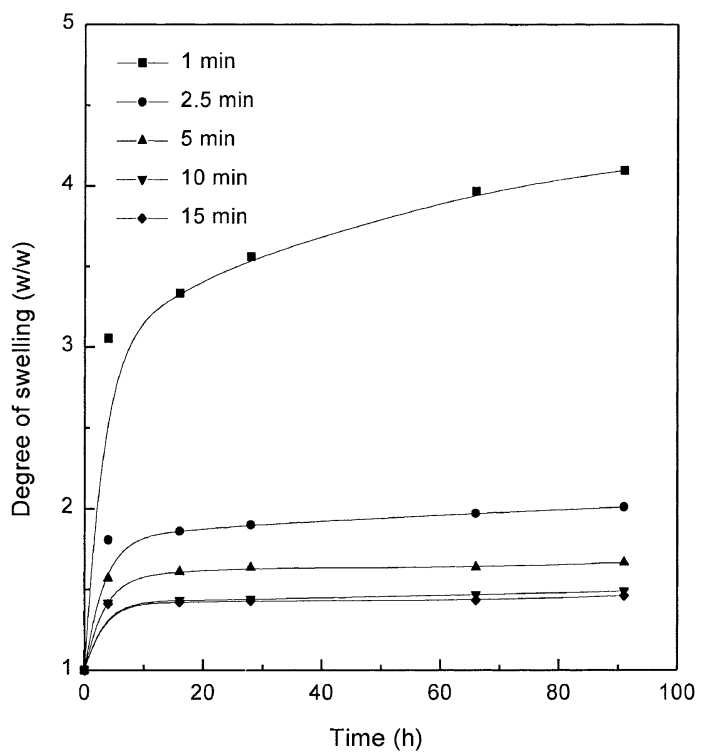

Figure 6. The degree of swelling of a sample SE115 in water at various times. The sample had been treated with different immersion times in a $0.25 \%$ glutaraldehyde solution.

branes. When soaked in the water, the outer chitosan shell absorbs a lot of water and swells greatly, which reduces the mechanical strength of the wet membranes. Some of the membranes even disintegrate when they are pulled out from the water. Therefore, these chitosan-graft-poly(vinyl acetate) copolymers were cross-linked with glutaraldehyde to increase their mechanical strength and acid resistance. The reaction of amino group in chitosan with glutaraldehyde forms an imine $(-\mathrm{C}=\mathrm{N}-)$ cross-link between linear chitosan chains. ${ }^{20}$ The extent of cross-linking is easily understood by measuring the degree of swelling of sample membranes. Figure 6 shows the degree of swelling of a sample SE115, which had been treated in a $0.25 \%$ glutaraldehyde with different soaking times. As the soaking time increased, the degree of swelling decreased, and consequently the extent of cross-linking increased. After $10 \mathrm{~min}$ in the glutaraldehyde solution, the swelling behavior was almost the same indicating the extent of cross-linking reached a plateau value. Table III lists the tensile mechanical properties of these wet membranes with various extents of cross-linking. The ultimate tensile strength and initial modulus increased with the extent of cross-linking, yet the elongation at break decreased. Figure 7 is the surface morphology of the wet membrane SE115 treated with a 10min immersion in the glutaraldehyde solution. Though a particulate membrane was still observed, voids were no longer found among the particles. It became a dense membrane due to the cross-linking. The effect of crosslinking on the copper ion adsorption is shown in Figure 8 , which a generalized diffusion process was assumed in the entire adsorption process. It is obvi- 
Table III. Initial modulus (E), ultimate tensile strength (UTS) and elongation at break (\%) of a SE115 wet membrane treated with different immersion times in a $0.25 \%$ glutaraldehyde solution

\begin{tabular}{lcccccc}
\hline Time/min & 0.5 & 1.5 & 2.5 & 5 & 10 & 15 \\
\hline$E / \mathrm{MPa}$ & 1.11 & 2.69 & 3.49 & 4.07 & 5.24 & 5.26 \\
& $\pm 0.08^{*}$ & \pm 0.07 & \pm 0.12 & \pm 0.07 & \pm 0.09 & \pm 0.08 \\
UTS/MPa & 0.35 & 0.68 & 0.75 & 0.81 & 0.90 & 1.16 \\
& \pm 0.03 & \pm 0.01 & \pm 0.01 & \pm 0.01 & \pm 0.07 & \pm 0.17 \\
Elongation/\% & 31.8 & 25.7 & 20.7 & 18.5 & 16.7 & 14.0 \\
& \pm 0.4 & \pm 1.3 & \pm 1.1 & \pm 0.6 & \pm 1.1 & \pm 0.6 \\
\hline
\end{tabular}

*Standard deviation.

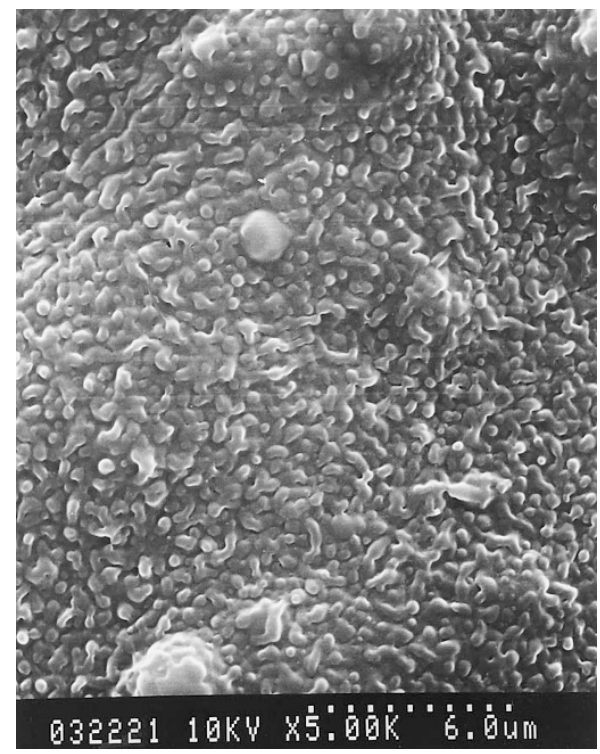

Figure 7. Surface micrograph of the SE 115 wet membrane after 10 -min immersion in a $0.25 \%$ glutaraldehyde solution.

ous that the diffusion rate and adsorption quantity decreased with the extent of cross-linking. It has been shown that the imine group is still capable of chelating with the copper ion as the amino group, ${ }^{21}$ yet its ability is smaller than the latter. In addition, the crosslinking has reduced the chain relaxation and flexibility, which increases the steric hindrance of the chelating reaction. Most importantly, the cross-linking has greatly decreased the porosity among the particles. Because of all these reasons, the adsorption of copper ion thus decreased.

\section{CONCLUSIONS}

Poly(vinyl acetate) was grafted onto the chitosan to increase its toughness and water resistance. A stable dispersion system was observed during the reaction as the chitosan molecules provided the stability of dispersed particles. Because of the rigid chitosan chains at the particles' surface, particulate membranes were formed after drying the solution. A two-stage behavior was observed when the adsorption of copper ion was carried out in an acid solution. The first stage was gov-

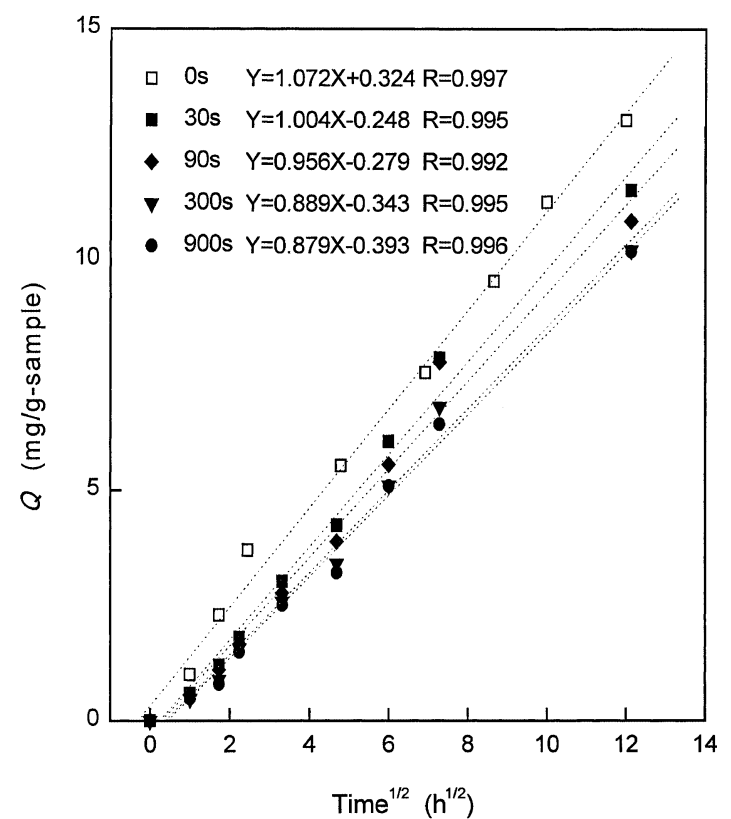

Figure 8. A linear plot of the specific adsorption quantity $Q$ of copper ion with the square root of time $t, Q=k t^{1 / 2}$. The sample SE115 had been treated with different immersion times in a $0.25 \%$ glutaraldehyde solution.

erned by the shell diffusion and reaction, whereas the second stage adsorption was controlled by the core diffusion. The mechanical strength of wet membranes can be improved by the cross-linking with the glutaraldehyde. Yet, this decreased the specific adsorption quantity of copper ion.

Acknowledgments. The authors wish to express their appreciation for the financial support of the National Science Council, Republic of China (Project No: NSC 89-2313-B-131-002-A24).

\section{REFERENCES}

1. K. Aoi, A. Takasu, and M. Okada, Macromolecules, 30, 6134 (1997).

2. K. Inoue, K. Yoshizuka, and K. Ohto, Anal. Chim. Acta, 388, 209 (1999).

3. Y. Kawamura, M. Mitsuhashi, and H. Tanibe, Ind. Eng. Chem. Res., 32, 386 (1993).

4. E. Guibal, C. Milot, and J. M. Tobin, Ind. Eng. Chem. Res., 37, 1454 (1998).

5. K. Inoue, Y. Baba, and K. Yoshiguza, Bull. Chem. Soc. Jpn., 66, 2915 (1993).

6. G. L. Rorrer, T. Y. Hsien, and J. D. Way, Ind. Eng. Chem. Res., 32, 2170 (1993).

7. T.-M. Don, C.-F. King, and W.-Y. Chiu, The Synthesis and Properties of Chitosan-Modified Poly(vinyl acetate), J. Appl. Polym. Sci., (in press).

8. G. Odian and J. H. T. Kho, J. Macromol. Sci. Chem., A4, 317 (1970).

9. K. H. Kim, K. S. Kim, and J. S. Shin, Polymer (Korea), 11, 
133 (1987).

10. T. Graczyk and V. Hornof, J. Polym. Sci., Part A: Polym. Chem., 26, 2019 (1988).

11. T. Graczyk, J. Macromol. Sci. Chem., A27, 23 (1990).

12. R. F. Storey and L. J. Goff, Macromolecules, 22, 1058 (1989).

13. M. J. Fernandez, I. Casinos, and J. M. Guzman, J. Polym. Sci., Part A: Polym. Chem., 28, 2275 (1990).

14. Y.-R. Chen, Master Thesis in Chemical Engineering, National Taiwan University, Taipei, Taiwan, 1997. p 35.

15. K. Toei and T. Kohara, Anal. Chim. Acta, 83, 59 (1975).

16. M.-L. Tsai, Ph. D. Dissertation in Department of Food Science, National Taiwan Ocean University, Keelung, Taiwan.
1997, p 17.

17. E. Onsoyen and Q. Skaugrud, J. Chem. Technol. Biotechnol., 49, 396 (1990).

18. C.-F. King, Master Thesis in Materials Science and Engineering, National Taiwan University, Taipei, Taiwan, 2000, p 62.

19. G. M. Barrow, "Physical Chemistry", 6th ed, McGraw-Hill, World Scientific Publishing Co. Pte. Ltd., Singapore, chapt. 17, 1996, pp 839-840.

20. T.-Y. Hsien and G. L. Rorrer, Ind. Eng. Chem. Res., 36, 3631 (1997).

21. T.-Y. Hsien and G. L. Rorrer, Sep. Sci. Technol., 30, 2455 (1995). 